\title{
Symposium discusses glass hybrid technology
}

Despite the EU-wide phase down of amalgam having been in operation for over 12 months, many practitioners are still searching for a viable alternative to amalgam that can be used without clinical compromise. As a result, leading manufacturer GC has taken the opportunity to bring together some of Europe's dental heavyweights to consider the most suitable alternatives for the postamalgam era.

The GC Symposium, held during the CED-IADR/NOF Oral Health Research Congress in Madrid in September, was chaired by Prof Avijit Banerjee from the Faculty of Dentistry, Oral \& Craniofacial Sciences, at King's College London and was entitled 'Finding the true alternative to amalgam with glass hybrid technology'.

Over 120 delegates were treated to a variety of presentations from some of Europe's leading restorative practitioners who outlined the specific criteria required for an appropriate amalgam alternative and then looked in more detail at the materials that best meet the immediate clinical challenges.

Prof Dr Marco Ferrari from the Department of Medical Biotechnologies at the University of Siena, looked at the clinical indications of glass hybrid materials, their physical and mechanical properties, clinical application and expected longevity. He underlined the many advantages of glass hybrids for both clinicians and patients which

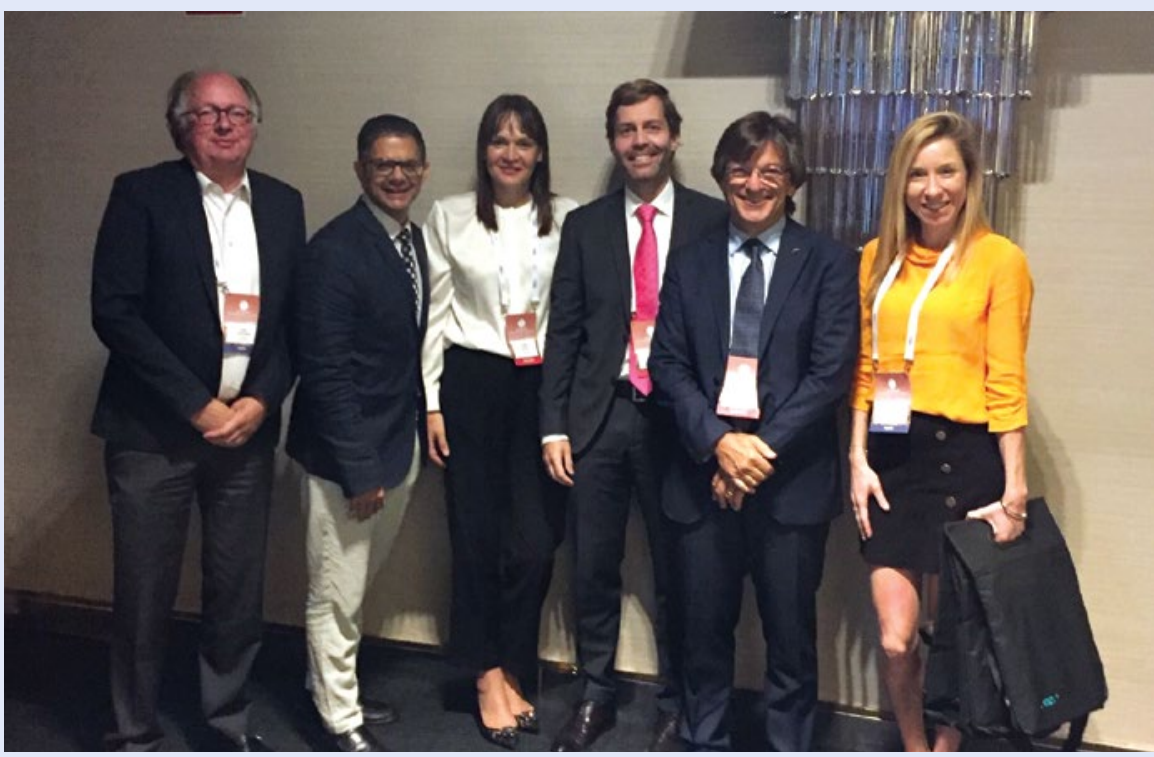

From left to right: Bart Dopheide (GC), Prof Dr Avijit Banerjee, Prof Dr Ivana Miletić, Prof Dr Falk Schwendicke, Prof Dr Marco Ferrari, Dr Cristina Parise Gre (GC)

include cost-efficiency, ease of handling and durability.

Prof Dr Ivana Miletić from the Department of Endodontics and Restorative Dentistry at the School of Dental Medicine, University of Zagreb, showed the results of her two-year multicentre clinical study with EQUIA Forte. She reported that this glass hybrid material had demonstrated similar clinical performance as nanohybrid composites after two years in moderate to large two surface (Class II) cavities. This study is now continuing into its third year, with early results that look equally as promising.

Launched in 2014, EQUIA Forte, and more latterly Equia Forte HT, both from GC, possess the key benefits that made amalgam such an important material of the past. Easy to handle, this unique glass hybrid material enables practitioners to create durable restorations that can be placed with speed and efficiency, enabling them to provide best quality care to all of their patients.

If you are searching for a viable alternative to amalgam, contact GC UK on 01908 218999, email info.uk@gc.dental or visit www.gceurope.com/products/ equiaforteht/ for more information on the latest glass hybrid technology.

\section{Implants that stay put}

Trycare Ltd is the UK Distributor for Adin Dental Implant Systems, the world's tenth largest manufacturer of dental implants. Clinical tests in universities and hundreds of thousands of successful restorations make Adin implants first choice for many implantologists.

Adin's spiral design implants are suitable for all indications and exceptional for immediate loading. Their surfaces have been treated for faster osseointegration and New York University research indicates that they integrate as well as Nobel, Straumann and Astra.
Their unique thread and tip slices through bone compressing and condensing on the way, offering outstanding primary stability and optimal restorative orientation. Whilst their surface treatment has been shown to increase osseointegration. Macroscopic grooves on the surface of the implant thread double the surface area of most other implants and give better bone-to-implant contact.

Adin spiral design implants can be redirected during placement with little or no reduction of stability, the dual cutting blade edge helping to minimise the osteotomy site and trauma to bone and surrounding soft tissue. This makes them ideal for thinner bone ridges.

There will be an opportunity to learn more about Adin implants at the Trycare September 2020 Extravaganza, London, 11 September. The speakers are world renown Patrick Palacci, Ulf Nannmark, Dr Paul Schuh and Marcos White from the UK. The event will deliver six hours of enhanced CPD for just $£ 395.00$ plus vat.

For further information visit the Trycare website, www.trycare.co.uk/adin, or request one of their Adin Catalogues.

To request a catalogue contact your local representative or call 01274885544 . 